\title{
QU'EST-CE QUE LE SLOVÈNE ET LE FRANÇAIS ONT EN COMMUN ? LES ARTICLES DÉFINI ET INDÉFINI
}

\section{INTRODUCTION}

La catégorie du défini et de l'indéfini occupe un chapitre à part dans la grammaire slovène. La raison en est avant tout le décalage entre les langues écrite et parlée. Dans la langue écrite (ou standard), le défini s'exprime généralement par l'utilisation de la forme définie de l'adjectif. Les substantifs sont considérés comme définis a priori, les verbes connaissent des formes conjuguées ou définies et indéfinies, etc. C'est précisément l'utilisation de la forme définie de l'adjectif qui pose le plus de difficultés aux locuteurs natifs du slovène.

La désinence - $i$ a pour ainsi dire disparu de la majorité des échanges familiers et a été remplacée par l'article $t a$. En raison de l'absence de relation entre les discours parlé et écrit, les écarts sont fréquents dans la langue écrite [...]. En outre, la différence entre les langues parlée, où le défini est codifié à l'aide d'un article, et écrite est si grande que les locuteurs natifs hésitent quant à la forme à employer s'ils n'ont pas appris ce point durant leur scolarité (Schlamberger Brezar 2004 : 42-43).

Si nous concentrons notre attention sur l'analyse du défini dans la langue parlée spontanée (par exemple celle des locuteurs de Ljubljana), nous remarquons que celle-ci est saturée d'actualisateurs généraux, c'est-à-dire d'articles définis et indéfinis. L'analyse précise des textes les plus divers montre ainsi que la langue parlée spontanée (des locuteurs de Ljubljana) exprime le plus souvent le défini et l'indéfini à l'aide d'actualisateurs généraux, ce qui, d'une certaine manière, la rapproche de nombreuses langues romanes et germaniques où l'usage des articles défini et indéfini est codifié dans le système même de la langue, mais l'éloigne de la langue écrite standardisée. De ce fait, bien souvent les locuteurs natifs du slovène hésitent au moment de choisir la forme de l'adjectif (définie ou indéfinie) qu'ils doivent utiliser (s'ils n'ont pas bien appris ce point auparavant). ${ }^{1}$

\section{LES ACTUALISATEURS TEXTUELS}

Les articles défini et indéfini appartiennent à la catégorie des actualisateurs textuels dont la fonction principale consiste à transformer la valeur lexicale du lexème qu'ils précèdent en référence concrète (signification) ou en orientation vers le caractère concret/non concret ou général de la référence présentée. Ainsi, à l'intérieur même du texte, l'indéfini et le défini sont identifiés précisément en raison de leur opposition soulignée par les différences entre les actualisateurs au moyen desquels ils s'expriment (voir : Vidovič Muha 1996: 115-116).

* Adresse de l'auteur : Filozofska fakulteta, Oddelek za slovenistiko, Aškerčeva 2, 1000 Ljubljana, Slovénie. Mél: mojca.rogac@guest.arnes.si

1 «/N/ous pourrions nous demander si le codage de la catégorie du défini dans la langue écrite des locuteurs natifs du slovène, tel qu'il est décrit dans les grammaires et manuels de slovène, $a$ encore valeur de système » (Schlamberger Brezar 2004: 43). 
Il s'agit de distinguer des éléments provenant de la relation entre l'indéfinitude propre au système linguistique et la définitude propre à la parole, des éléments provenant donc d'une relation intralinguistique, du « langage ». Pour dénommer cette relation, nous pouvons avoir recours à l'hypéronyme définitude linguistique. Comme nous l'avons déjà dit, cette relation se base sur l'indéfinitude propre au système linguistique - sémantisme lexical (virtualité) et la définitude propre à la parole. Dans des textes concrets, cette définitude peut se réaliser soit comme définitude soit comme indéfinitude, les deux étant bien entendu intratextuelles, susceptibles d'être déterminées au niveau de la définitude de la parole sur la base de leur opposition mutuelle (définitude textuelle / indéfinitude textuelle) et discriminables grâce à la différence de leurs supports - actualisateurs. (Vidovič Muha 1996: 115-116)

La principale fonction des actualisateurs étudiés est donc l'expression de la catégorie sémantique de mise en situation spatio-temporelle.

Du point de vue du sens, cette information est définie de trois manières différentes : par son contenu ou sa qualité ( " comprehension »), par son extension dans l'espace, qui lui fournit des éléments de quantifications, et par son rapport spatial (et temporel) envers moi, mes discours et mes interlocuteurs (Mikuš 59/60: 11).

La fonction des actualisateurs peut ainsi être assumée par différents moyens linguistiques. Ils se départagent en deux principaux groupes : les actualisateurs généraux et les actualisateurs spécifiques. Sont classés dans le groupe des actualisateurs généraux les articles définis et indéfinis et dans celui des actualisateurs spécifiques les noms propres (qui ne sont dotés de leur valeur informative qu'une fois utilisés dans le texte), les syntagmes nominaux (dont sont exclues les locutions phraséologiques constitutives du dictionnaire), les pronoms et les numéraux.

\section{$\underline{\text { Schémas : Les actualisateurs textuels }}^{2}$}

Actualisateurs textuels

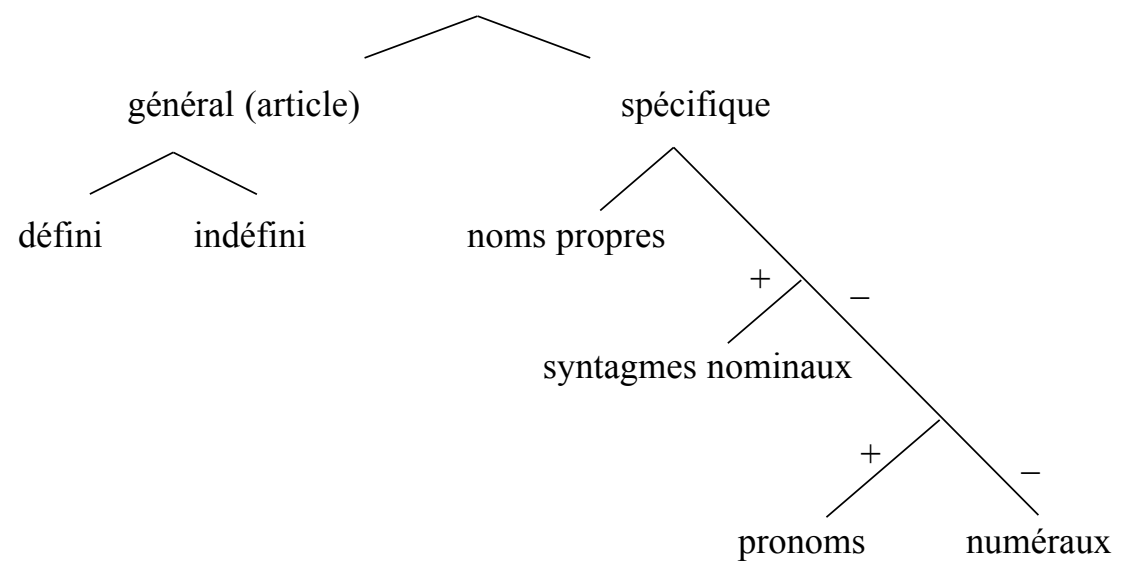

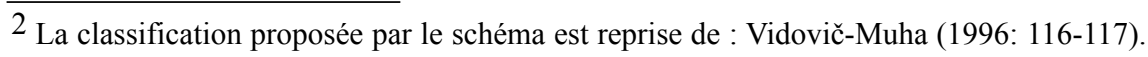


La différence fondamentale entre les actualisateurs textuels généraux et spécifiques réside dans le fait que les premiers sont porteurs des trois éléments actualisateurs (le contenu, la quantité et l'espace/temps). Cela signifie que l'article est en lui-même asémantique et peut ainsi à la fois transmettre et dévoiler des éléments actualisateurs qui caractérisent, quantifient et localisent. À l'opposé des actualisateurs généraux, les actualisateurs spécifiques soulignent explicitement un seul des éléments actualisateurs tandis qu'ils établissent avec les deux autres une relation implicite.

\subsection{En et $t a$}

Dans la région centrale de la Slovénie, ce sont surtout en et ta qui assument la fonction d'actualisateurs textuels généraux (dans le discours spontané).

Exemples :

$\mathrm{Ja}$, ne, to je tko kot, a veš, sta se vidla čez akvarij, a veš, un film Romeo in Julija, a veš, v tej ta zadni verziji, $\mathrm{k}$ je Leonardo Dicaprio, no tam je ena scena, $\mathrm{k}$ se vidta čez akvarij (B12/45).

Dans cet exemple, l'adjectif démonstratif et l'article défini sont utilisés conjointement. Le premier est déclinable ( $v$ tej verziji) et entre en relation avec le noyau substantival. Du point de vue fonctionnel, il se réalise avant tout au niveau de la référence à la réalité extralinguistique. Le second $t a$ (article) n'entre pas en relation avec le noyau substantival ; il est le signe que l'adjectif qu'il précède est déterminé (ta zadni).

Tam je vse tko mrtvo, žive duše ni, vsi so že šli, turisti, več al manj zgleda, da domov, ne midva z Nastjo sva rekla, sva mogla še tle za Samota ta star televizor dol prinest, pol pa, ne vem, kva je še naroču (B11/37).

Ici aussi, la fonction du lexème « ta » est très nette. C'est le signe du catactère défini de l'adjectif qu'il précède.

Pol je, pol je ob pol devetih je bil Boštjan že tam. Pride en, a je to stažist, al kaj jaz vem kaj, en mlad dohtar, k še niti ni dohtar, mislim, da je stažist, ne, pa tud če ne, no: »Ja, bomo kar mehur prepiknil, ne« (B1/5).

In pol reče: »Ja ja, zdaj bomo pa mi še nekam šli. No, kam naj gremo?«, ne, a veš, pol smo šli še v en kafič, tko k ga je Janez poznal in je Janez kar mislu, da bomo tam cel večer zabil (B2/7).

Dans les deux exemples, l'article en est le signe de l'indéfini, le signe annonçant que le locuteur ne développera pas le lexème qui suit l'actualisateur (il ne fournira pas de renseignements complémentaires).

Si nous observons tous les exemples cités du point de vue distributif, nous pouvons conclure (peut-être un peu hâtivement) que l'article indéfini est lié du point de vue fonctionnel au substantif (les substantifs expriment l'indéfini à l'aide de l'article 
en/ena/eno), tandis que l'article défini est lié à l'adjectif (les adjectifs expriment le défini à l'aide de l'article $t a$ ). Ce que nous venons d'écrire pourrait prouver que les articles défini et indéfini en slovène suivent une évolution indépendante et ne sont en aucun cas un germanisme.

Dans de tels exemples, en réalité nous éprouvons donc le besoin d'utiliser un article défini, et c'est toujours ce que fait le peuple slovène. A-t-il repris cette habitude des Allemands ? Non, car il ne l'utilise pas là où les Allemands l'utilisent, c'est-à-dire devant les substantifs ; il ne l'utilise que dans les cas où les anciens Slovènes l'utilisaient, selon les besoins de leur langue locale (Škrabec 1994: 210).

Si nous partons de cette citation, nous pouvons donc conclure que l'« interruption » de l'emploi des articles défini et indéfini chez Ravnikar ${ }^{3}$ est non fondée et que, d'une certaine manière, jusqu'à nos jours elle est à l'origine des hésitations des locuteurs natifs concernant l'usage correct/approprié de la forme définie de l'adjectif et l'expression du défini et de l'indéfini en général.

Dans les œuvres de Ravnikar (Sgodbe svetiga Pisma sa Mlade Ljudi, 1815), les articles défini et indéfini disparaissent de la langue standard écrite [...]. On doit sans aucun doute y voir dans une certaine mesure l'influence de Kopitar. Entretemps est parue sa grammaire qui, sur les plans phonétique, morphologique, syntaxique et lexical, a orienté la codification de la langue slovène écrite vers un purisme rigoureux dû au rapport à l'allemand, tout en accroissant l'attention portée aux dialectes slovènes, au vieux slave et aux autres langues slaves (Orožen 1972: 112).

\subsubsection{L'actualisateur ta}

En principe, le lexème $t a$ assume le rôle d'article défini uniquement quand il est mis en relation avec un adjectif. Dans ce cas, la forme est invariable ( $t a / t a / t a)$. Dans les textes (retranscrivant le discours spontané), on remarque souvent que l'actualisateur $t a$ précède aussi le substantif employé seul (sans adjectif épithète).

Exemple:

M: Skratka eno štorijo ti bom povedou o enmu profesorju fizike z oxfor- oxfordske univerze, $\mathrm{k}$ ga dobr puznam in $\mathrm{k}$ je tak ful štoser, in predlanskem je mel pač $\mathrm{v}$ enem letniku enga študenta, $s$ katerim se res ni zastopu, ne, in tud un ga ni maru, tko da je blo čist recipročno, ta ta ... in enkrat je bil spet en konflikt, v polni predavalnci je blo, ne, in kar naenkrat ta profesor udpre predal na svojem katedru, potegne ven pištolo in ga ustreli,

${ }^{3}$ Matevž Ravnikar (20.9.1776 - 20. 11. 1845) fut le fondateur de la chaire de langue slovène à la Faculté de théologie de 1'Université de Ljubljana. Il était également professeur de philosophie et enseignait la Bible. Son œuvre la plus connue est l'ouvrage intitulé Sgodbe svetiga Pisma sa Mlade Ljudi (Histoires de la Bible pour la jeunesse). 


\section{tega študenta, in ta študent ...}

$\mathrm{S}$ : A to je res, al kaj?

M: To je res, to je čist avtentična štorija, ta študent pade, raide mort, kot bi rekli v francoščini, in pač totalna panika v predavalnici, saj slišal »Oh, God, « a veš, pa »ka pa je zdaj to, saj se mu je čist strgal, « ne, in begat sem pa tja, da ne bi še kerga pihnu, ne, no, in čez kakšno minuto se pa ta študent lepo pobere, otrese prah s sebe in se usede nazaj kot da ni nič, in sta se pač očitno, klub temu da se po- čist zares nista marala, sta se zmenila za štos, k sta pač vedla, da vsi vejo, da se ne marata, in da bo to zlo avtentična šala, da bojo vsi verjel, in so dejansko cela predavalnca je padla na finto, ne, in panika, in vse, pa nič, ne, po so pa naprej mel predavanje. Ampak pištola je bla čist taprava, sam je bil slep naboj, logično. (B33/102)

Dans cet exemple, les lexèmes en in ta sont utilisés conjointement. Ils précèdent tous les deux des substantifs qui ne sont qualifiés par aucun adjectif ou autre déterminant. Il est intéressant de constater que les articles en et $t a$ sont reliés avant tout aux substantifs se rapportant aux personnages principaux du récit (ou le noyau du récit). Ce sont le professeur (profesor) et l'étudiant (študent). Lors de leur première occurrence, ils sont précédés de l'actualisateur en (bom povedou o enmu profesorju fizike z oxforoxfordske univerze [...] in predlanskem je mel pač v enem letniku enga študenta). Par la suite, ils sont systématiquement précédé par l'actualisateur ta (in kar naenkrat ta profesor udpre predal na svojem katedru, potegne ven pištolo in ga ustreli, tega študenta, in ta študent /.../ta študent pade).

Le locuteur a utilisé l'actualisateur indéfini également devant certains autres substantifs (eno štorijo, v enem letniku, en konflikt). Tous les trois sont mentionnés pour la première fois et le locuteur ne les complète pas, n'ajoute aucune précision les concernant ( $v$ enem letniku - inscrit dans une année d'études $\rightarrow$ peu importe laquelle). Tous les trois forment le noyau de la réalité constitutive de la proposition du texte ou du monde textuel.

En dehors des substantifs susmentionnés, précédés d'articles, nous rencontrons dans les textes onze autres substantifs qui ne sont précédés d'aucun article ou actualisateur : univerza, predavalnica, predal, kateder, pištola, prah, štos/šala, finta, panika, predavanje, naboj. Traduits en français, ces substantifs sont précédés d'un article : l'université, la salle de cours, le tiroir, la chaire, un pistolet, la poussière, la panique, le cours, une balle. L'absence d'article devant les substantifs énumérés précédemment nous dévoile partiellement les lois régissant l'utilisation des articles défini et indéfini dans la langue parlée spontanée des locuteurs de Ljubljana tels qu'on les rencontre dans l'exemple analysé.

Le substantif univerza - université est défini déjà à l'aide de l'épithète oxfordska - d'Oxford. De ce fait, il est impossible de le faire précéder d'un article indéfini ; dans le cas contraire, on pourrait comprendre qu'il existe plusieurs universités d'Oxford. L'utilisation des mots predavalnica, pištola et naboj dévoile complètement le caractère originellement défini des substantifs slovènes. L'utilisation de l'article indéfini ne serait possible que si l'adjectif épithète était mentionné (par exemple, dans une structure du type : $v$ eni polni predavalnici, potegne ven eno staro pištolo). Lors de la seconde mention du mot predavalnica (totalna panika v predavalnici), le substantif 
est déjà défini par le contexte textuel. Il a déjà été mentionné auparavant, mais, comme il ne participe pas du noyau de la proposition du texte, il serait inattendu de le faire précéder d'un article défini. Le substantif naboj est déjà déterminé par une épithète (slep naboj). Ici aussi, il est impossible de le faire précéder d'un article indéfini, parce qu'il n'y a eu qu'une seule balle - naboj - et que le mot est défini par le contexte textuel par la mention antérieure du pistolet - pištola. Et pourtant, malgré cela l'utilisation de l'article défini est impossible (*sam je bil taslep naboj). En effet, même si l'ordre des mots ne le confirme pas complètement, naboj assume la fonction de sujet et l'adjectif slep celle de prédicat. Le mot kateder est défini au niveau textuel par l'adjectif possessif qui le précède. L'usage de l'article défini serait redondant, inattendu : comme il ne fait pas partie du noyau de la proposition du texte, le substantif se trouverait particulièrement mis en évidence par la présence de l'article. Le mot predal dévoile, lui aussi, le caractère originellement défini des substantifs. L'article indéfini dans le texte pourrait nous faire comprendre que la chaire - kateder - comporte plusieurs tiroirs. L'article défini serait également inattendu, car predal ne représente pas le noyau de la proposition du texte.

Ayant une fonction englobante, les substantifs štos et finta ne pourraient être précédés que d'un article défini, et pourtant le locuteur l'a omis. Par ailleurs, la locution pasti na finto est figée et, de ce fait, ne prévoit pas l'utilisation d'un actualisateur.

Si nous résumons tout ce qui vient d'être énoncé, nous pouvons formuler les affirmations suivantes :

- L'article défini peut également être utilisé devant un substantif ou une locution substantivale.

- L'utilisation des articles défini et indéfini devant un substantif ou une locution substantivale et souvent liée aux personnages principaux du récit, aux personnes constitutives du noyau de la proposition du texte, c'est-à-dire les substantifs ou locutions substantivales présentant/caractérisant les personnes (personnages), les choses, les concepts sur lesquels se concentre le récit.

- Les personnages secondaires (également les noms d'objets et de concepts) sont généralement utilisés sans article (défini ou indéfini), ce qui confirme que les substantifs slovènes sont par définition définis.

- L'utilisation des articles défini et indéfini diffère assez de leur utilisation en français et en allemand. L'utilisation de l'article défini devant un substantif ou une locution substantivale en slovène est plus un procédé stylistique qu'un élément morphologique, ${ }^{4}$ puisque les formes peuvent être utilisées avec ou sans article.

- Souvent l'utilisation de l'article défini devant un substantif ou une locution substantive se recoupe partiellement avec d'autres fonctions métatextuelles ou discursives (par exemple, en tant que moyens anaphoriques ou cataphoriques, en tant que signifiant du rapport au contenu, etc.).

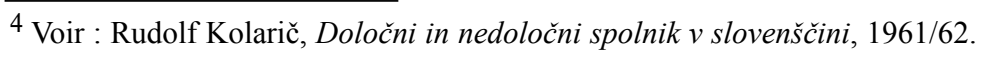


- L'utilisation de l'article défini devant un adjectif (par exemple, $v$ tej ta zadni, ta star) est morphosyntaxique : dans la langue parlée (des locuteurs de Ljubljana), le caractère défini ou indéfini de l'adjectif est encodé presque exclusivement à l'aide de l'article. Même lorsque l'adjectif précède le substantif et forme avec lui une locution substantivale ( $v$ tej ta zadni verziji, ta star televizor), l'article défini est relié à l'adjectif pour exprimer son caractère défini (il s'agit d'un phénomène morphosyntaxique) et, indirectement aussi, bien sûr, le caractère défini de la locution adjectivale au niveau textuel.

\subsection{Autres actualisateurs textuels}

En dehors des articles en et $t a$, on distingue dans le discours parlé spontané de nombreux autres actualisateurs que l'on pourrait appeler (de même que les deux articles généraux) des éléments métatextuels ou signifiants discursifs. Ce sont, par exemple : (en) tak, tist(i), un, nek, tale. Ils remplissent les fonctions les plus diverses, pouvant être des moyens anaphoriques ou cataphoriques, des déictiques, des marqueurs caractérisant le rapport au contenu, des éléments limitant la validité, des moyens interrompant l'axe linéaire, des déictiques secondaires, des éléments caractérisant l'univers commun, des éléments permettant l'expression du caractère défini ou indéfini au niveau textuel, etc.

Tabelau: Les fonctions textuelles et interpersonnelles de quelques actualisateurs textuels

\begin{tabular}{|c|c|c|c|c|c|c|c|c|}
\hline & \multicolumn{8}{|c|}{ Fonctions textuelles et interpersonnelles } \\
\hline $\begin{array}{l}\text { Actualisateu } \\
\text { rs textuels }\end{array}$ & $\begin{array}{l}\text { Fonction } \\
\text { anapho- } \\
\text { rique }\end{array}$ & $\begin{array}{l}\text { Fonction } \\
\text { catapho- } \\
\text { rique }\end{array}$ & $\begin{array}{l}\text { Fonction } \\
\text { déictique }\end{array}$ & $\begin{array}{l}\text { Fonction } \\
\text { de } \\
\text { catracté- } \\
\text { risation } \\
\text { du rap- } \\
\text { port au } \\
\text { contenu }\end{array}$ & $\begin{array}{l}\text { Fonction } \\
\text { limitant } \\
\text { la validité }\end{array}$ & $\begin{array}{l}\text { Fonction } \\
\text { interrom- } \\
\text { pant l'axe } \\
\text { linéaire }\end{array}$ & $\begin{array}{l}\text { Fonction } \\
\text { de déic- } \\
\text { tique } \\
\text { secon- } \\
\text { daire. }\end{array}$ & $\begin{array}{l}\text { Fonction } \\
\text { de } \\
\text { caracté- } \\
\text { risation } \\
\text { de l'uni- } \\
\text { vers com- } \\
\text { mun }\end{array}$ \\
\hline (en) $t a k$ & $*$ & $*$ & $*$ & $*$ & $*$ & & $*$ & \\
\hline tisti & * & $*$ & $*$ & $*$ & * & $*$ & $*$ & \\
\hline un & $*$ & $*$ & $*$ & $*$ & & $*$ & $*$ & $*$ \\
\hline nek & & & & * & $*$ & & & $*$ \\
\hline tale & * & $*$ & $*$ & $*$ & & $*$ & & * \\
\hline
\end{tabular}

La plupart des actualisateurs étudiés assument plusieurs fonctions à la fois. Par exemple, l'actualisateur tist (i) peut agir sur le plan fonctionnel comme un moyen cataphorique et anaphorique. Il peut également caractériser le rapport du locuteur au contenu (le locuteur dévoile son opinion subjective concernant ce qui est dit), il peut limiter la validité, ce qui permet locuteur d'exprimer ses doutes concernant le carac- 
tère approprié ou inapproprié du lexème choisi ou bien concernant l'appellation donnée au lexème. Il peut aussi être un procédé interrompant l'axe linéaire ou un déictique secondaire, lorsqu'il transporte hic et nunc le monde textuel éloigné dont parle le locuteur. Enfin, il peut également caractériser l'univers commun quand le locuteur exprime ainsi la conviction que le récepteur sait de quoi il parle et qu'il n'est pas nécessaire de lui expliquer le sens du lexème que l'actualisateur précède.

\section{Exemple:}

Recimo, no, recimo, je to taka lušna, $\mathrm{k}$ je bil Val majčkn, saj to boste vi tud vidl, takrat $\mathrm{k}$ bo Leon mal zrastu, za ene tolk, k je bil že kar ene dve tri leta star, in enkat sedi tko na kahlci, ne, in jaz... to v Martulku, ne, in jaz mu kupim en Kinder jajček, je že takrat norel za temi Kinder jajčki, ne, in on je spet to reku Kindel solplajs, in je skratka ... sedi na kahlci pa prav »:Dida, odvi mi,« ne, jaz pa v tisti naglc, mislim, da sem hotu prpravt mu tist njegov zrezek, ne, k je stalno meso jedu, ne, rečem »: Joj, Val, ti bom pol, ne, rajši, ne. Zdele nimam časa.« Pa prav »: Ne, kar odvi.« In sem mogu odvit, ne, potem je pa reku »: Ja, « čez neki časa, seveda tam notr je tist jajček, ne, čokoladnega itak on ni nikol pojedu, ga je kar zraven odložu, pa tko naprej, ne, nakar je tist jajček..., je reku »: Ja, pa tud udpri, ne.« No, po sem pa še tist, sem se udal v usodo, ne, sem reku, zdaj bo dal mir, k bo vidu, kaj je notr, kakšna stvar, ne, kaj za sestavt, al, ne vem, ne, in skratka, je po tem no, grem jaz nazaj h tisti svoji kuhi, ne, nakar on prav »: Dida, na zdaj mi pa puvej, kako se to sestav, bova sestavla, ne.« Jaz sem reku »: Val, veš kaj, nimam špeglov tuki, ne.« Potem se pa zlo zamisl, pa neki časa je tih, potem pa prav, ko gleda tist listek, ne, na kterm je blo pač shema za sestavt tale tisto igrco v Kinder jajčku oziroma tist tist predmetek, ne, in prav »: Dida, tuki piše: Ne jabiš očal.« (B34/103)

Dans cet exemple, le locuteur utilise à huit reprises l'actualisateur tist/a. Au niveau fonctionnel, les emplois de l'actualisateur étudié diffèrent les uns des autres. Le premier tisti ( $v$ tisti naglci) souligne la signification du mot avec lequel il entre en relation, mais, en même temps, il est le signe de l'éloignement temporel de l'événement (il s'agit d'une action passée) dont parle le locuteur. Le deuxième tisti (tist njegov zrezek) souligne explicitement la signification du mot auquel il se rapporte. L'adjectif possessif njegov a également pour fonction de souligner et de mettre en valeur le substantif. Ensemble, ils expriment donc une action répétitive et évidente, la préparation de l'escalope (priprava zrezka). Le troisième tisti (tist jajček) exprime un fait connu de tous qu'il dévoile et souligne à l'aide de la particule modale seveda. Le quatrième tisti assume au moins deux fonctions conjointement. Il joue un rôle anaphorique et est ainsi l'élément qui permet de remonter le temps ; en même temps, il est l'élément qui met en valeur le sens du mot avec lequel il entre en relation. Le cinquième tisti/a (h tisti svoji kuhi) est sur le plan fonctionnel proche du deuxième actualisateur tisti. Avec le possessif, il souligne le sens du mot auquel il se rapporte. En même temps, nous pouvons affirmer qu'il joue aussi un rôle anaphorique (1. sem hotu prpravt mu tist njegov zrezek $\rightarrow 2$. grem jaz nazaj h tisti svoji kuhi). En dehors de souligner le sens du mot qu'il précède, le sixième tisti (tist listek) indique égale- 
ment que nous avons affaire à une réalité objective connue de tout le monde ou (selon le locuteur) connue de son interlocuteur. Sur le plan du sens, les septième et huitième tisti/a (tisto igrco, tist predmetek) peuvent être interprétés par la périphrase "saj veste katero/katerega ». Sur le plan fonctionnel, ils sont donc proches du troisième actualisateur tisti par lequel le locuteur exprime la conviction que son interlocuteur sait de quoi il parle et qu'il est inutile de lui expliquer le sens du lexème précédé par l'actualisateur.

Nous pouvons clore cet examen attentif de l'emploi de l'actualisateur tist/a en concluant que l'actualisateur étudié précède, lui aussi, le plus souvent les personnages principaux du récit, c'est-à-dire ceux qui constituent le noyau de la proposition du texte.

\section{Conclusion}

L'expression de la détermination, et plus spécialement de la détermination des adjectifs, cause des difficultés même aux locuteurs natifs avertis de la langue slovène. La désinence -i pour exprimer le défini dans la langue parlée spontanée (surtout dans la région centrale de la Slovénie) est remplacée par l'article $t a$. On peut se demander, s'il est vraiment justifié d'affirmer que l'article ta, évincé il y a 150 ans, est inacceptable en slovène standard ou soutenu. ${ }^{5}$ La problématique étudiée mérite incontestablement de faire l'objet d'un examen plus approfondi fondé tant sur des faits historiques et sociolinguistiques que sur les caractéristiques morphosyntaxiques contemporaines.

Dans les textes retranscrivant le discours direct spontané, l'article $t a$ intervient aussi devant les substantifs et locutions substantivales non précédés d'un adjectif épithète ou autre déterminant. L'usage est le plus souvent lié aux éléments du texte qui constituent le noyau de la proposition du texte. Souvent, il se recoupe aussi avec les autres fonctions métatextuelles ou discursives (par exemple, celle de moyen anaphorique ou cataphorique, celle d'élément caractérisant le rapport au contenu ou au récepteur, celui d'élément caractérisant l'univers commun, etc.). En tout cas, nous pouvons conclure que l'utilisation de l'actualisateur ta précédant le substantif seul est un moyen stylistique dépendant pour une large part de nombreux facteurs conditionnant l'acte discursif.

\section{SOURCES}

34 documents enregistrés rapportant le discours oral de locuteurs s'exprimant dans le cadre d'une conversation privée. Une fois retranscrits, ces enregistrements comprennent au total 148427 signes (espaces non comprises). Tous les locuteurs sont originaires de Ljubljana ou de ses environs.

\footnotetext{
${ }^{5}$ Le slovène écrit standard a sans raison rejeté cet article défini. Maintenant que l'on distingue à l'intérieur de la langue standard la langue soutenue et la langue familière, nous pouvons dire que, dans la langue familière, les formes avec $t a$ alternent assez librement avec les formes sans $t a$. Pour la langue soutenue, ce $t a, 150$ ans plus tard, n'est pas acceptable (il ne peut apparaître que dans des citations, comme une particularité stylistique dans quelque conte populaire ; Finžgar l'utilise dans ses contes pour souligner le caractère populaire du discours) (Toporišič 1978: 299).
} 


\section{BIBLIOGRAPHIE}

KOLARIČ, Rudolf (1961/62) « Določni in nedoločni spolnik v slovenščini. » Jezik in slovstvo 5/2, 40-44.

MIKUŠ, Radivoj F. (1959/60) «Prostorni podatak događaja : teorija i govorni izraz. » Radovi, Razdio lingvističko - filološki 1. Zadar: Filozofski Fakultet.

MIKUŠ, Radivoj F. (1972) « Principes de syntagmatique » Paris: Didier.

OROŽEN, Martina (1972) « K določnemu členu v slovenščini. »Slavistična revija 20/14, 105-114.

SCHLAMBERGER BREZAR, Mojca (2004) « Diskurzivni pristop k problemu določnosti v slovenščini. » Jezik in slovstvo 49/5, 35-44.

SMOLEJ, Mojca (2006) Vpliv besedilne vrste na uresničitev skladenjskih struktur (primer narativnih besedil v vsakdanjem spontanem govoru). Thèse de doctorat. Ljubljana: Filozofska fakulteta.

ŠKRABEC, Stanislav (1994) « Nekoliko slovenške slovnice za poskušnjo. »In : Jezikoslovna dela 2 (XIV. tečaj, 3. zvezek). Nova Gorica: Frančiškanski samostan Kostanjevica, 208-210.

TOPORIŠIČ, Jože (1978) «Imenska določnost v slovenskem knjižnem jeziku. » Slavistična revija 26/3, 287-303.

VIDOVIČ MUHA, Ada (1996) « Določnost kot besedilna prvina v slovničnem opisu slovenskega jezika (ob Kopitarjevi slovnici). » In : Jernej Kopitar in njegova doba, 115-130.

\section{Résumé \\ QU'EST-CE QUE LE SLOVÈNE ET LE FRANÇAIS ONT EN COMMUN ? LES ARTICLES DÉFINI ET INDÉFINI}

On peut distinguer deux principaux groupes d'actualisateurs textuels : les actualisateurs généraux et les actualisateurs spécifiques. Le premier groupe comprend les déterminants définis et indéfinis, tandis que le second est constitué des noms propres, des locutions substantivales, des pronoms et des numéraux.

Le slovène standard exprime le défini, entre autres, grâce à la forme définie de l'adjectif (dans les locutions figées, après les adjectifs démonstratifs et possessifs, quand il s'agit d'évoquer une particularité déjà connue ou évoquée, etc.). De leur côté, les substantifs sont en principe toujours définis. Cependant, si nous analysons l'expression du défini et de l'indéfini dans la langue parlée spontanée (par exemple, des locuteurs de Ljubljana), nous pouvons remarquer que cette dernière se différencie souvent de la langue standard par la présence généralisée des actualisateurs généraux, autrement dit des déterminants définis et indéfinis. L'analyse précise de textes variés montre ainsi que la langue parlée spontanée des locuteurs donnés exprime le plus souvent le défini et l'indéfini à l'aide d'actualisateurs généraux, ce qui, d'une certaine manière, la rapproche des langues romanes et germaniques où l'expression du défini et de l'indéfini est déjà codifiée dans le système de la langue standard. 


\section{Povzetek \\ KAJ IMATA SLOVENŠČINA IN FRANCOŠČINA SKUPNEGA? DOLOČNI IN NEDOLOČNI ČLEN}

Besedilni aktualizatorji se delijo na dve večji skupini, na skupino globalnih aktualizatorjev in skupino specialnih aktualizatorjev. V skupini splošnih so določni in nedoločni členi, v skupini posebnih pa so lastna imena, imenske zveze, zaimki in števniki.

Knjižna slovenščina določnost izraža npr. z določno pridevniško obliko (v stalnih zvezah, za vrstnimi kazalnimi in svojilnimi zaimki, ko gre za znano oz. že omenjeno lastnost...), samostalniške besede pa so načeloma tako in tako določne. Če se osredotočimo na analizo določnosti v spontano govorjenem jeziku (npr. govorcev Ljubljane), lahko opazimo, da se le ta pogosto razlikuje od knjižnega jezika, saj je v njem prisotnih vse polno globalnih aktualizatorjev oz. določnih in nedoločnih členov. Natančna analiza najrazličnejših besedil tako pokaže, da spontano govorjeni jezik (npr. govorcev Ljubljane) določnost oz. nedoločnost največkrat izraža prav z globalnimi aktaulizatorji, kar ga na nek način približuje številnim romanskim in germanskim jezikom, kjer je raba določnega in nedoločnega jezika zakodirana v sam sistem knjižnega jezika. 